\title{
CD38 Deficiency Protects Heart from High Fat Diet-Induced Oxidative Stress Via Activating Sirt3/FOXO3 Pathway
}

\author{
Ling-Fang Wang ${ }^{a} \quad$ Cong-Cong Huang ${ }^{a} \quad$ Yun-Fei Xiao ${ }^{a, b} \quad$ Xiao-Hui Guan ${ }^{a}$ \\ Xiao-Nv Wang ${ }^{\mathrm{a}}$ Qing Cao ${ }^{\mathrm{a}}$ Yu Liu ${ }^{\mathrm{a}}$ Xuan Huang ${ }^{\mathrm{a}}$ Li-Bin Denga \\ Ke-Yu Deng ${ }^{a}$ Hong-Bo Xin ${ }^{a, b}$ \\ Institute of Translational Medicine, Nanchang University, Nanchang, ${ }^{b}$ College of Life Sciences, \\ Nanchang University, Nanchang, China
}

\author{
Key Words \\ Cd38 • Oxidative stress $\bullet$ Heart .Oleic acid $\bullet$ Sirt3/FOXO3 pathway
}

\begin{abstract}
Background/Aims: Previous studies showed that CD38 deficiency protected heart from ischemia/reperfusion injury and high fat diet (HFD)-induced obesity in mice. However, the role of CD38 in HFD-induced heart injury remains unclear. In the present study, we have investigated the effects and mechanisms of CD38 deficiency on HFD-induced heart injury. Methods: The metabolites in heart from wild type (WT) and CD38 knockout (CD38 ${ }^{-1-}$ ) mice were examined using metabolomics analysis. Cell viability, lactate hydrogenase (LDH) release, super oxide dismutase (SOD) activity, reactive oxygen species (ROS) production, triglyceride concentration and gene expression were examined by biochemical analysis and QPCR. Results: Our results revealed that $\mathrm{CD} 38$ deficiency significantly elevated the intracellular glutathione (GSH) concentration and GSH/GSSG ratio, decreased the contents of free fatty acids and increased intracellular $\mathrm{NAD}^{+}$level in heart from $\mathrm{CD} 38^{-/-}$mice fed with HFD. In addition, in vitro knockdown of CD38 significantly attenuated OA-induced cellular injury, ROS production and lipid synthesis. Furthermore, the expression of mitochondrial deacetylase Sirt3 as well as its target genes FOXO3 and SOD2 were markedly upregulated in the $\mathrm{H} 9 \mathrm{C} 2$ cell lines after OA stimulation. In contrast, the expressions of NOX2 and NOX4 were significantly decreased in the cells after OA stimulation. Conclusion: Our results demonstrated that CD38 deficiency protected heart from HFD-induced oxidative stress via activating Sirt3/FOXO3-mediated antioxidative stress pathway.




\section{Introduction}

Over-nutrition leads to metabolic-related changes in hearts including lipid accumulation and mitochondrial disturbances [1]. Lipid accumulation in cardiomyocytes is greatly correlated with lipotoxic effects on the heart including diastolic dysfunction [2] and increased oxidative stress [3]. Oxidative stress causes impaired cardiac fatty acid metabolism and endoplasmic reticulum (ER) stress, and induces other severe adverse effects such as insulin resistance [4]. In addition, overweight and obesity result in excessive activation of cardiac renin-angiotensin system [5], which further facilitate NADPH activation and ROS production. Therefore, the altered myocardial lipid metabolism may have a causative role in metabolic heart diseases.

CD38 (also known as cyclic ADP ribose hydrolase) is a membrane glycoprotein that plays important roles in cell proliferation, muscle contraction, hormone secretion and immune responses [6]. Specifically, CD38 acts as a multifunctional ectoenzyme to catalyze the synthesis of cyclic ADP-ribose (cADPR) from $\mathrm{NAD}^{+}$and hydrolysis of cADPR to ADPribose (ADPR) [7-9]. Cyclic ADPR plays an important role in $\mathrm{Ca}^{2+}$ release from $\mathrm{Ca}^{2+}$ stores [10], whereas its main product, ADPR, can be covalently attached to proteins such as TRPM2, $\mathrm{a} \mathrm{Ca}^{2+}$-permeable cation channel, by which it also modifies $\mathrm{Ca}^{2+}$ signaling $[11,12]$. Meanwhile, CD38 can also catalyze NADP ${ }^{+}$to NAADP, which is a potent trigger for $\mathrm{Ca}^{2+}$ mobilization [13]. In addition, CD38 can modulate the activity of sirtuins, a family of the NAD-dependent deacetylases that had beneficial effects in life span, mitochondrial function and energy metabolism in mammalian cells $[14,15]$.

In mammals, the sirtuin family comprises seven proteins (Sirt1-Sirt7), which are categorized as class III histone deacetylases. Sirt3 is expressed in a variety of tissues, especially in mitochondria-rich tissues such as brain, liver and heart tissues [16]. Sirt3 regulates global protein acetylation in mitochondria to control oxidative metabolism via using mitochondrial $\mathrm{NAD}^{+}$as a substrate [17]. Many studies showed that Sirt3 played important roles in many diseases including cardiac and neurodegenerative diseases $[18,19]$. Dysfunction of mitochondria is associated with production of ROS [20]. It has been reported that high levels of free fatty acid can induce oxidative damage, whereas lowering the levels of free fatty acid can reduce oxidative stress in hepatocytes [21]. Moreover, elevated activity of NAPDH Oxidase 2 (NOX2) increases palmitate-induced mitochondrial ROS production in cardiomyocytes [22]. In contrast, Sirt3 attenuates ROS production by activating the transcription factor FOXO3a, and then trans-activating antioxidant genes such as manganese superoxide dismutase (MnSOD) and catalase in $\mathrm{NAD}^{+}$-dependent manner [23-25]. Moreover, the upregulation of the expression and activity of Sirt3 increases SOD2 activity via deacetylation of the lysine residues, and then reduces mitochondrial oxidative stress and intracellular ROS production $[26,27]$.

It has been reported that the intracellular levels of $\mathrm{NAD}^{+}$were increased in various tissues from CD38 deficient mice [8]. Chini et al. demonstrated that CD38 deficiency protected mice from HFD-induced obesity [28]. Our previous studies showed that mouse embryonic fibroblasts (MEFs) isolated from CD38 knockout mice were resistant to oxidative stress via inhibiting ROS production and $\mathrm{Ca}^{2+}$ overload [29] and CD38 deficiency significantly enhanced cardiac functions in male mice [30]. Recently we observed that CD38 deficiency protected heart from ischemia/reperfusion injury via activating anti-oxidative stress pathway and inhibiting $\mathrm{Ca}^{2+}$ overload [31]. Moreover, our previous study showed that CD38 deficiency moderately improved the cardiac function in Ang-II induced cardiac hypertrophy [32]. However, the role of CD38 in HFD-induced heart injury is not evaluated. In the present study, we observed the metabolic changes of CD38-deficient heart, which may be associated with CD38 deficiency-mediated resistance to HFD-induced oxidative stress. In addition, we observed the significant changes of redox homeostasis, cofactor generation and lipid metabolism in CD38-deficent heart compared with the heart from wild type mice. Knockdown of CD38 significantly reduced lipid-induced oxidative damages in H9C2 cardiomyocytes. 


\section{Cellular Physiology Cell Physiol Biochem 2018;48:2350-2363 \begin{tabular}{ll|l} 
DOI: 10.1159/000492651 & $\begin{array}{l}\text { O 2018 The Author(s). Published by S. Karger AG, Basel } \\
\text { www.karger.com/cpb }\end{array}$
\end{tabular}}

Wang et al.: CD38 Deficiency Suppresses Oxidative Stress

\section{Materials and Methods}

\section{Animal experiments}

Eight week-old, male CD38\% mice and the genetics- and age-matched wild-type male mice (C57BL/6 background) were used in the experiments. CD38 $\%$ mice were obtained from Dr. Frances E. Lund (Rochest). The mice were maintained under controlled temperature $\left(22-24^{\circ} \mathrm{C}\right)$ and illumination $(12$-h dark/light cycle) with free access of water. All animals were treated in accordance with the Guide for the Care and Use of Laboratory Animals, and approved by the Ethics Committee of Nanchang University. Mice were fed with normal diet or high-fat diets (60\%-HFD, D12492; Research Diets Inc.) for 12 weeks. Then, the mice were anesthetized by $4 \%$ chloral hydrate and hearts were dissected and rinsed with phosphate-buffered saline and then immediately stored at $-80^{\circ} \mathrm{C}$ for further experiments.

\section{Metabonomic analysis}

Hearts isolated from WT and CD38 $\%$ mice were prepared for metabonomic analysis using the automated MicroLab STAR® system from Hamilton Company. After removing protein, the samples were loaded on Waters ACQUITY ultra-performance liquid chromatography (UPLC) and a Thermo-Finnigan LTQ mass spectrometer (LC-MS) or analyzed on a Thermo-Finnigan Trace DSQ fast-scanning single-quadrupole mass spectrometer.

\section{Cell Culture and transfection}

H9C2 cells (American Type Culture Collection, Manassas, USA) were cultured in DMEM (Thermo Fisher, Waltham, MA, USA) supplemented with $10 \%$ fetal bovine serum (FBS, Gibco Ltd., Gaithersburg, USA), $100 \mathrm{U} / \mathrm{ml}$ penicillin and $100 \mathrm{mg} / \mathrm{ml}$ streptomycin at $37^{\circ} \mathrm{C}$ in an atmosphere of $5 \% \mathrm{CO}_{2}$. $\mathrm{H} 9 \mathrm{C} 2$ stable cell line with CD38 knocking-down was prepared in our laboratory as previously described [31].

\section{Oleic acid stimulation}

Oleic acid (Sigma-Aldrich) stock solution was prepared in PBS with 20\% BSA. This solution was diluted to $0.5 \mathrm{mM}$ Oleic acid solution with DMEM. The medium with only BSA was used as control. H9C2 cells were incubated with $\mathrm{OA}(0.5 \mathrm{mM})$ for $24 \mathrm{~h}$ before analysis.

\section{Cell viability assay}

H9C2 cells were seeded into 96-well plates and incubated at a density of $1 \times 10^{4}$ cells/well for $24 \mathrm{~h}$. The cells were treated with $0.5 \mathrm{mM}$ Oleic acid (OA) or BSA for $6 \mathrm{~h}$ and $12 \mathrm{~h}$, respectively. Cell viability was detected with cell counting kit-8 (CCK8) from Dojindo, Japan. H9C2 cells were treated with CCK8 at $37^{\circ} \mathrm{C}$ for $3 \mathrm{~h}$, the absorbance of $450 \mathrm{~nm}$ was measured using a microplate reader to quantify the formazan products. The amount of the formazan dye generated by dehydrogenases in cells was directly proportional to the number of living cells.

\section{Measurements of $L D H$ and SOD activities}

LDH and SOD activities were detected using Cytotoxicity LDH Assay Kit-WST and SOD Assay Kit-WST (Dojindo, Mashiki-machi, Japan) according to the manufacturer's protocols, respectively. Briefly, cells (2x $10^{5} /$ well) were seeded into 6-well plates and treated with $0.5 \mathrm{mM} \mathrm{OA}$ or BAS for $24 \mathrm{~h}$. For LDH activity assay, culture supernatant was collected and centrifuged at $1000 \mathrm{rpm}$ for $5 \mathrm{~min}$. Supernatant was taken, mixed with Working Solution in 96-well plate at room temperature for $20 \mathrm{~min}$, and then added Stop Solution to each well, then immediately measured the absorbance at $490 \mathrm{~nm}$ by a microplate reader. For SOD activity assay, the cells were washed with PBS. The cells were lysed by ultrasonication. The samples were centrifuged at $10000 \times \mathrm{g}$ for $10 \mathrm{~min}$ at $4^{\circ} \mathrm{C}$. The supernatant was used for SOD activity (relative activity) determination according to the instruction. Absorbance was measured at $450 \mathrm{~nm}$ using a microplate reader. All experiments were performed at least three times. 


\section{Cellular Physiology Cell Physiol Biochem 2018;48:2350-2363 \begin{tabular}{ll|l} 
and Biochemistry Published online: 15 August, 2018 & $\begin{array}{l}\text { (c) } 2018 \text { The Author(s). Published by S. Karger AG, Basel } \\
\text { www.karger.com/cpb }\end{array}$ \\
\hline
\end{tabular}}

ROS detection

The intracellular ROS production was measured using H2DCF-DA (Sigma-Aldrich). Firstly, $2 \times 10^{5}$ / well H9C2 cells were incubated with $10 \mu \mathrm{M}$ H2DCF-DA for $30 \mathrm{~min}$. Then the cells were washed twice with PBS and collected for detection. Then the fluorescent dichlorofluorescein was monitored with automatic microplate reader at $488 \mathrm{~nm}$ excitation wavelength and $525 \mathrm{~nm}$ emission wavelength. All procedures should be kept away from light.

Quantitative Real-Time PCR analysis

Cells $\left(2 \times 10^{5} /\right.$ well $)$ were plated in 6 -well plate and treated with $0.5 \mathrm{mM} \mathrm{OA}$ or BAS for $24 \mathrm{~h}$. Total RNA from cells were isolated using the Trizol method (Thermo Fisher) followed by DNase treatment. RNA content was measured by Nano Drop 2000 (Thermo Fisher). Then RNA was reversely transcribed using the Takara high capacity cDNA synthesis kit (TaKaRa, Dalian, China) according to the instruction. Relative expression of mRNAs was determined after normalized to GAPDH using the $\triangle \mathrm{Ct}$ method. Quantitative PCR was performed using the ABI-ViiA7 PCR machine. The sequences of the primers are listed below: CD38, forward 5'-CTGCCAGGATAACTACCGACCT-3' and reverse 5'-CTTTCCCGACAGTGTTGCTTCT-3'; Sirt3, forward 5' ${ }^{\prime}$-CCCAATGTCGCTCACTACTT-3' and reverse $5^{\prime}$-AGGGATACCAGATGCTCTCT-3'; SOD2, forward 5'-GGACAAACCTGAGCCCTAA-3' and reverse 5'-GCGACCTTGCTCCTTATTG-3'; FOXO3, forward 5'- CCTGTCCTACGCTGACCTGAT-3' and reverse 5'-AGTCCCTTCGTTCTGAACCCG-3'; NOX4, forward 5'-AGTCAAACAGATGGGATA-3' and reverse 5'-TGTCCCATATGAGTTGTT-3'; Bax, forward 5'-GGGTGGCAGCTGACATGTTT-3' and reverse 5'-GCCTTGAGCACCAGTTTGC-3'; Bcl2, forward 5'-GTTGCAGTCACCGGATTCCT-3' and reverse 5'-CGGA GGTGGTGTGAATCCA-3'; GAPDH, forward 5'-AGCCAAAAGGGTCATCATCT-3'; reverse 5'-GGGGCCATCCACAGTCTTCT-3'.

\section{Western blot analysis}

H9C2 cells were cultured in 100-mm culture dishes at a density of $1 \times 10^{6}$ cells $/$ dish. After treated with $0.5 \mathrm{mM} \mathrm{OA}$ or BAS for $24 \mathrm{~h}$, the cells were lysed with RIPA buffer ( $1 \mathrm{mM}$ PMSF was added) on ice for $30 \mathrm{~min}$, and centrifuged at $12000 \times \mathrm{g}$ for $10 \mathrm{~min}$ at $4^{\circ} \mathrm{C}$. Protein concentration was determined with Pierce BCA Protein Assay Kit (Thermo Fisher Scientific, Rockford, USA). Lysates then subjected to SDS-PAGE electrophoresis, transferred to PVDF membrane (Millipore), and probed with the primary antibodies against CD38 (Santa cruz), FASN (Abcam), Acetylated-Lysine (CST), Sirt3 (CST), SOD2 (CST), FOXO3 (Abcam), NOX2 (Abcam), NOX4 (Abcam), Bax (CST) and GAPDH (Abcam). The bound antibodies were detected by secondary HRPconjugated antibodies and the bands were visualized using the ECL system (Thermo Fisher Scientific, USA). Band images were obtained by using Molecular Imager ChemiDoc XRS+ (Bio-Rad, Hercules, CA, USA) and the intensity was analyzed by Image Lab $^{\text {TM }}$ software version 5.1 (Bio-Rad).

\section{Statistical analysis}

All data were presented as mean \pm SEM. The data were represented for three independent experiments. The statistical analyses were performed using SPSS19.0 software. The data were compared using Student's t test or one-way ANOVA. Differences were considered significant at $p<0.05$.

\section{Results}

Deficiency of CD38 leads to significant changes of the global metabolomics of hearts

Although metabolic abnormity has been widely explored in many tissues with obesity, little is known about the global metabolic changes of the hearts in HFD condition. We used LCMS and GC-MS to analyze metabolic fingerprints in hearts from CD38 deficient and wild type mice fed with HFD for 12 weeks. Principal component analysis and hierarchical clustering revealed a distinct difference in the two groups of samples, in which it may reflect significant metabolic shifts due to absence of CD38 (Fig. 1A-B). In addition, after peak detection, 271 known metabolites were acquired, and 87 of them were significantly altered $(p<0.05)$, including 27 metabolites were upregulated and 60 metabolites were downregulated. In addition, there were 17 compounds reaching statistically to a significant $(0.05<p<0.1)$. An estimation of the false discovery rate (q-value) is calculated to take into account the multiple 


\section{Cellular Physiology Cell Physiol Biochem 2018;48:2350-2363

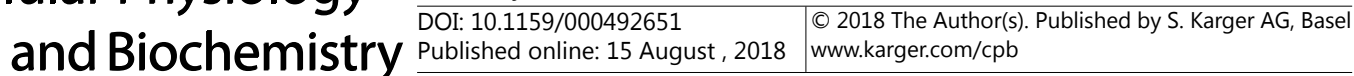 \\ Wang et al.: CD38 Deficiency Suppresses Oxidative Stress}

Fig. 1. CD38 deficiency leads to significant changes of the metabolites in hearts. The metabolite levels in heart tissue from wild type and CD38 knockout mice were determined by Principal component analysis (PCA) (A) and unsupervised hierarchical clustering analysis (B) of metabolomics assay $(n=5)$. The red represents the elevated metabolites and the green represents the depressed metabolites. The relative concentrations of nicotinamide adenine dinucleotide (NAD) and $\mathrm{NAD}^{+}$precursor nicotinamide mononucleotide (NMN) in hearts from CD38 knockout and wild type mice (C). The relative levels of CD38 metabolites including nicotinamide and ADP-ribose in hearts from CD38 knockout and wild type mice (D). The concentrations of GSH and GSSG were measured in heart tissues from wild type and CD38 knockout mice (E). The GSH/GSSG ratios were determined in hearts from CD38 knockout and wild type mice (F). Data are shown as means \pm SEM, ${ }^{* *} \mathrm{p}<0.01, \mathrm{n}=$ 5 per group.

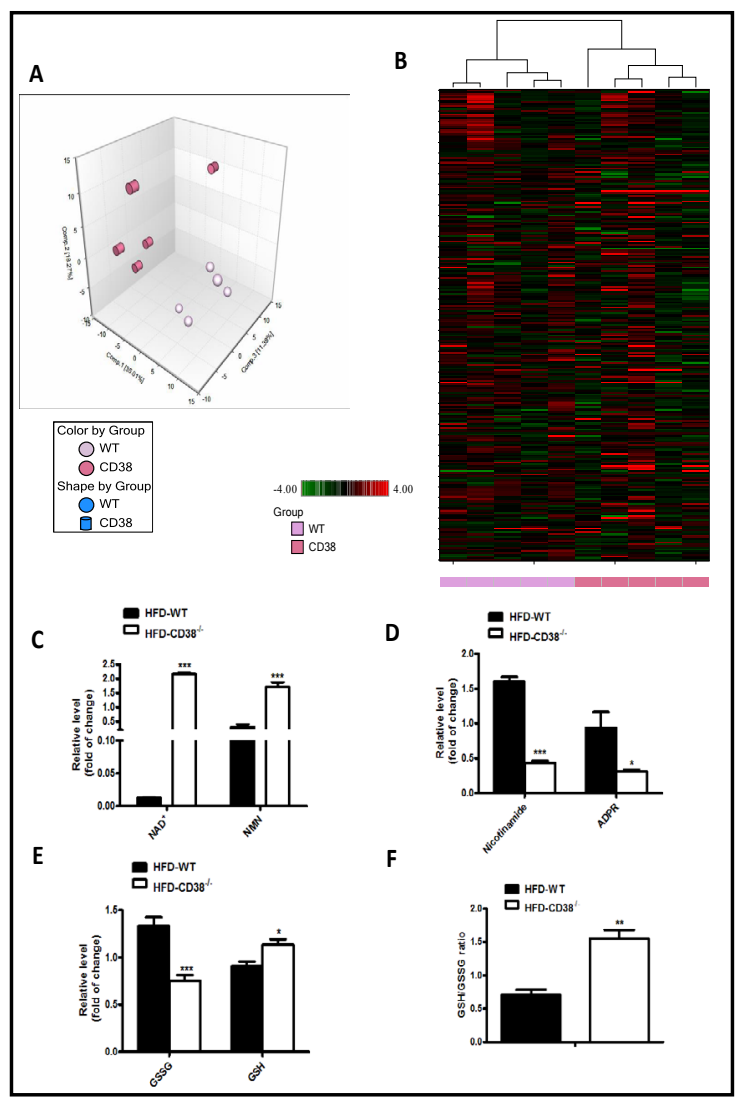

comparisons that normally occur in metabolomic-based studies. A q-value threshold of $<0.05$ was used to remove any p-values (up to a 95\% confidence) that could have been false positives.

CD38 deficiency leads to marked changes of $\mathrm{NAD}^{+}$metabolism including the increased intracellular GSH concentration and GSH/GSSG ratio in the heart of mice fed with HFD

Among the altered metabolites, we observed that there were significant changes in $\mathrm{NAD}^{+}$ metabolism in CD38 deficient hearts. The CD38 substrate $\mathrm{NAD}^{+}$level was elevated in CD38 deficient heart (Fig. 1C). In addition, $\mathrm{NAD}^{+}$precursor nicotinamide mononucleotide (NMN) was also increased (Fig.1C). However, the metabolites of CD38 including nicotinamide and ADP-ribose were significantly reduced in CD38 deficient hearts compared with those in WT group (Fig.1D). Furthermore, we found that two substances glutathione (GSH) and oxidized glutathione (GSSG) that were closely related to the oxidative stress were significantly changed in hearts of CD38 deficient mice compared to WT counterparts. The level of GSH in CD38 deficient heart was remarkably increased compared with those in WT, while GSSG was significantly decreased (Fig. 1E). Therefore, the GSH/GSSG ratio in CD38 deficient heart $(1.55 \pm 0.29)$ was remarkably increased compared with those $(0.70 \pm 0.18)$ in WT mice fed with HFD ( $p<0.01$; Fig. 1F). These results indicated that CD38 deficiency protected hearts from HFD-induced oxidative stress.

\section{Knockdown of CD38 protects from Oleic acid-induced cell death and oxidative stress}

In order to investigate the protective effects of CD38 deficiency on lipid-induced oxidative stress in vitro, we observed the effects of CD38 deficiency on oleic acid-induced oxidative stress in cardiomyocytes using CD38 knockdown H9C2 stable cell lines. First, we examined CD38 expression in CD38 knockdown H9C2 stable cell lines. As expected, CD38 mRNA level was approximately decreased by $97 \%$ in the knocking-down stable cell lines (Fig. 


\section{Cellular Physiology Cell Physiol Biochem 2018;48:2350-2363 \begin{tabular}{l|l|l} 
DOI: 10.1159/000492651 & $\begin{array}{l}\text { O 2018 The Author(s). Published by S. Karger AG, Basel } \\
\text { www.karger.com/cpb }\end{array}$ \\
\cline { 2 - 4 }
\end{tabular}

Fig. 2. CD38 knockdown protects cardiomyocytes from oleic acid-induced cell death and oxidative stress. The CD38 mRNA (A) and protein (B) expressions were determined by q-PCR and western blot in the CD38 knockdown stable H9C2 cell lines and normal H9C2 cells (cardiomyocytes). Survival rates of cardiomyocytes were detected by CCK8 assay in CD38 knockdown H9C2 cells or control cells after incubation with $0.5 \mathrm{mM}$ oleic acid for $6 \mathrm{~h}$ (left) and 12 (right) hours (C). The LDH (D) and SOD (E) activities in the supernatant of the cell culture after oleic acid stimulation were determined by measuring the absorbance (OD) at $490 \mathrm{~nm}$ and $450 \mathrm{~nm}$, respectively, in CD38 knockdown H9C2 cells or normal cells. The mean fluorescence intensities of the OA-induced ROS production were quantitatively analyzed with different groups of cells (F). Data are shown as means \pm SEM, ${ }^{*} \mathrm{p}<0.05,{ }^{* *} \mathrm{p}<0.01$ and ${ }^{* * *} \mathrm{p}<0.001$, $\mathrm{n}=3$ per group.

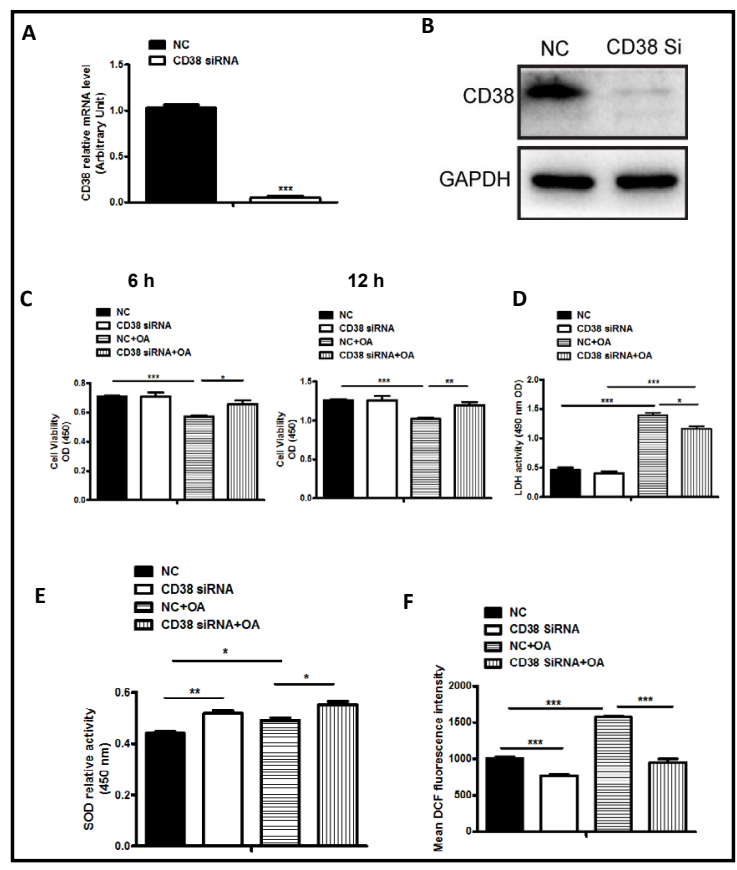

2A). In addition, the CD38 protein level was also significantly downregulated in the CD38 knockdown H9C2 cells (Fig. 2B). After incubation with $0.5 \mathrm{mM}$ oleic acid for 6 and 12 hours, the cells viability was determined using CCK8. As shown in Fig. 2C, the cell viability was significantly decreased in CD38 siRNA group compared with control siRNA group after OA stimulation for 6 and 12 hours, suggesting that CD38 knockdown might protect cells against cytotoxicity from oleic acid. Moreover, the results also showed that OA remarkably increased LDH activity and knockdown of CD38 significantly reduced LDH release in cardiomyocytes (Fig. 2D). In addition, the results showed that CD38 knockdown cells were resistant to OAinduced impairment of SOD activity in cardiomyocytes (Fig. 2E). Importantly, we found OA significantly increased ROS production in control cells, while CD38 knockdown reduced ROS generation (Fig. 2F). Taken together, these results indicated that CD38 deficiency protected cardiomyocytes from $\mathrm{OA}$-induced oxidative stress in vitro.

\section{CD38 deficiency significantly decreases lipid synthesis in vivo and in vitro}

Besides GSH and GSSG, we also found that metabolites involved in lipid synthesis were changed in CD38 deficient heart compared with WT under HFD. Lipid overload was one of causes resulting in oxidative damage. In CD38 deficient heart, we observed that most of free fatty acids were significantly decreased compared with WT mice. As shown in Fig. 3A, all of the long chain fatty acids including stearate (18:0), nonadecanoate (19:0), eicosenoate (20:1) and arachidate (20:0) were reduced in CD38 deficiency group. Moreover, palmitate (16:0) and oleate (18:1n9) were also decreased although there was no statistical difference. And the polyunsaturated fatty acids (n3 and n6), such as linoleate (18:2n6), linolenate [alpha or gamma; $(18: 3 \mathrm{n} 3$ or 6$)]$, docosadienoate $(22: 2 \mathrm{n} 6)$ and dihomo-linoleate $(20: 2 \mathrm{n} 6)$ were also decreased in CD38 deficient heart tissue (Fig. 3B). Moreover, we also observed that there was a significant decrease in lipid deposition in CD38 knockdown H9C2 cells treated with oleic acid through Oil Red 0 staining (Fig. 3C). And we also found that knockdown of CD38 significantly decreased intracellular TG level in H9C2 cells induced by oleic acid (Fig. 3D). Importantly, CD38 knockdown decreased the expression of key lipogenic gene FASN in H9C2 cells treated with oleic acid (Fig. 3E-F). These results suggested that decreased lipid synthesis in CD38 deficient heart and CD38 knockdown cardiomyocytes may reduce lipidinduced oxidative stress. 
Fig. 3. CD38 deficiency leads to significant decreases of lipid synthesis in vivo and in vitro. The free fatty acids including long chain fatty acid (A) and polyunsaturated fatty acid (B) were quantitatively determined in hearts from CD38 knockout and wild type mice. The lipids of cardiomyocytes were detected by Oil red 0 staining in CD38 knockdown H9C2 cells treated with or without oleic acid (C). The intracellular TG levels were quantitatively measured in CD38 knockdown H9C2 cells under OA stimulation (D).The western blot images (E) and quantitative analysis (F) of the protein level of the lipid synthetic gene FASN were determined in $\mathrm{H} 9 \mathrm{C} 2$ cells. Data are shown as means $\pm \mathrm{SEM},{ }^{*} \mathrm{p}<0.05,{ }^{* *} \mathrm{p}<0.01$ and ${ }^{* * *} \mathrm{p}<0.001, \mathrm{n}$ $=3-5$ per group.
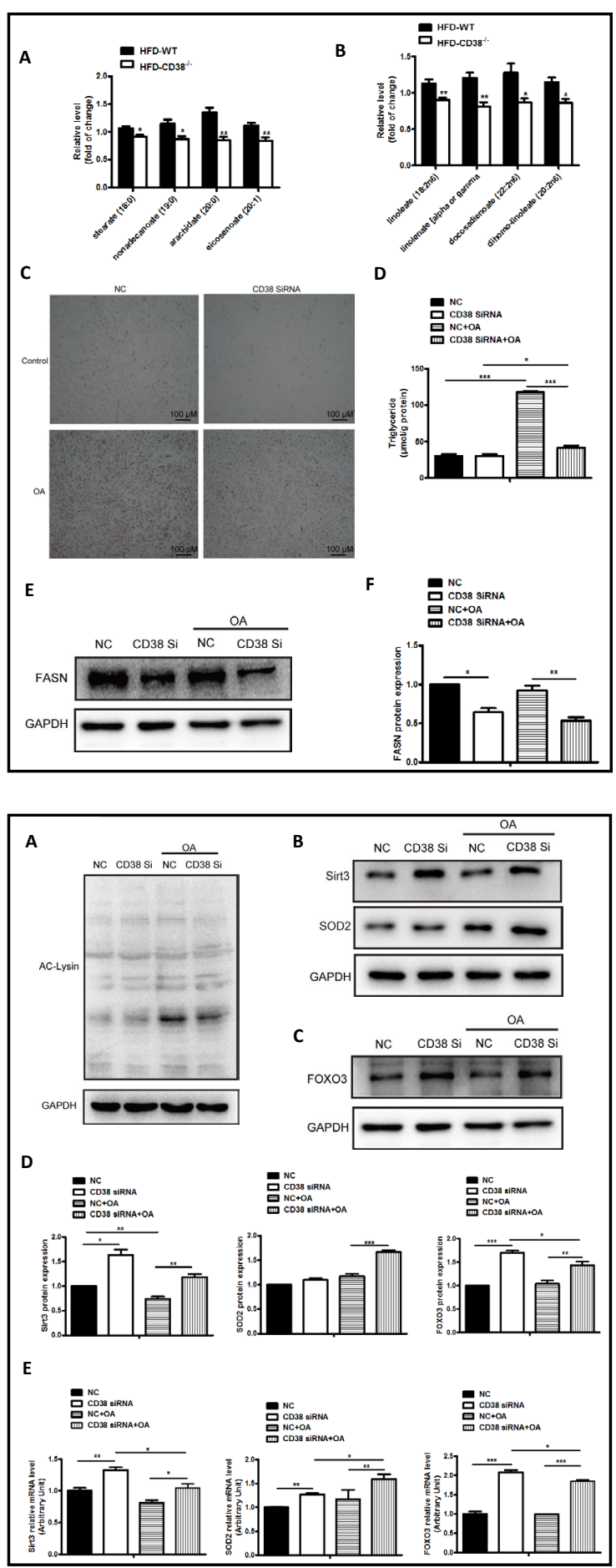

E
Fig. 4. CD38 knockdown increases the expressions of Sirt3, FOXO3 and SOD2 in oleic acid-treated cardiomyocytes. The protein acetylation levels of CD38 knockdown H9C2 cells with or without OA were analyzed by western blotting with antibody against acetylated-lysine (A). The expressions of Sirt3 (B), SOD2 (B) and FOXO3 (C) protein were determined by western blot analysis in CD38 knockdown H9C2 stable cells with or without oleic acid stimulation. The protein levels of Sirt3, SOD2 and FOXO3 were quantified from three different experiments (D). The mRNA expressions of Sirt3, SOD2 and FOXO3 were determined by qPCR (E). The values represent the means \pm SEM, ${ }^{*} \mathrm{p}<0.05$, ${ }^{* *} \mathrm{p}<0.01$ and $^{* * *} \mathrm{p}<0.001, \mathrm{n}=3$ per group.

Knockdown of CD38 promotes the expressions of Sirt3, FOXO3 and SOD2 in Oleic acidtreated cardiomyocytes

As shown in Fig. 1C, the level of $\mathrm{NAD}^{+}$was increased in CD38 deficient heart tissue. Sirt3 is a NAD+-dependent deacetylase which is mainly localized in mitochondria, and it deacetylates a number of mitochondrial proteins including manganese superoxide dismutase, which is responsible for limiting the accumulation of reactive oxygen species (ROS)[26]. In order to assess whether the mechanism of the protection form OA-induced oxidative stress was involved in the CD38/Sirt3 pathway, we first examined the total protein 
acetylation levels. The results showed that $\mathrm{OA}$ increased the level of protein acetylation and CD38 knockdown decreased it compared with control group under OA stimulation (Fig. 4A). Moreover, the mRNA and protein level of Sirt3 was also examined by RT-qPCR and Western blot analyses, respectively. CD38 knockdown dramatically increased the expression of Sirt3 at both protein (Fig. 4B and 4D) and mRNA levels (Fig. 4E) in OA stimulated cells. In addition, the results showed that the expression of SOD2, one of the Sirt3 target genes, was also upregulated in both OA stimulated and vehicle treated CD38 knockdown H9C2 cells, especially under the OA stimulation (Fig. 4B, 4D and 4E). In addition, our results showed that the expression of FOXO3, another target gene of Sirt3 which also regulates SOD2 expression, was consistent with Sirt3 in CD38 knockdown cells (Fig. 4C-E). These findings suggested that CD38 deficiency protected cardiomyocytes from OA-induced oxidative stress through Sirt3-mediated activation of FOXO3/SOD2 signaling pathway.

Knockdown of CD38 attenuates the expressions of NOX2, NOX4 and Bax but increases Bcl2 expression in Oleic acid-treated cardiomyocytes

As NADPH oxidase was associated with ROS production, we next detected the effects of CD38 knockdown on the expression of NOX2 and NOX4 after OA stimulation. We observed that the expressions of NOX2 and NOX4 were decreased in CD38 knockdown cells at both protein and mRNA levels, especially the NOX4 (Fig. 5A, B). Furthermore, the expressions of the apoptotic-related proteins were markedly altered when the CD38 was knockdown, seen as the Bax expression was significantly decreased, whereas the Bcl-2 expression was markedly increased in response to OA stimulation (Fig. 5C-E). As showed in Fig. 5F, the ratio of Bax/Bcl2 was significantly decreased in CD38 knockdown H9C2 cells with or without oleic acid stimulation. The working model of protective effects of CD38 deficiency on HFDinduced heart injury was presented in Fig. 6.

Fig. 5. CD38 knockdown deceases the expressions of NOX2, NOX4 and Bax but increases Bcl2 expression in oleic acid-treated cardiomyocytes. The expressions of NOX2 and NOX4 protein were determined by western blot analysis with or without oleic acid stimulation in CD38 knockdown or normal H9C2 cells (A). The expressions of NOX4 mRNA were determined by qPCR with or without oleic acid stimulation (B). The expressions of Bax protein were determined by western blot analysis in CD38 knockdown or normal H9C2 cells with or without oleic acids (C).The mRNA expressions of $\mathrm{Bax}(\mathrm{D})$ and $\mathrm{Bcl} 2$ (E), and the ratio of $\mathrm{Bax} / \mathrm{Bcl} 2$ (F) were quantitatively determined by qPCR in CD38 knockdown or normal H9C2 cells with or without oleic acid stimulation. The values represent the means \pm SEM, ${ }^{*} \mathrm{p}<0.05,{ }^{* *} \mathrm{p}<0.01$ and ${ }^{* * *} \mathrm{p}<0.001$, $\mathrm{n}=3$ per group.

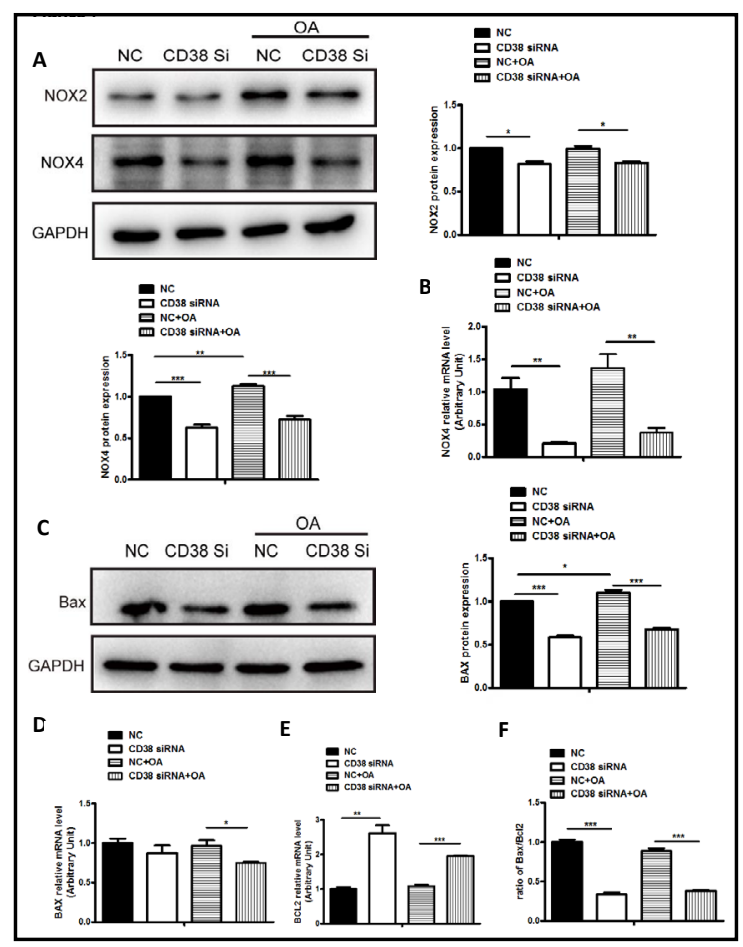




\section{Cellular Physiology Cell Physiol Biochem 2018;48:2350-2363

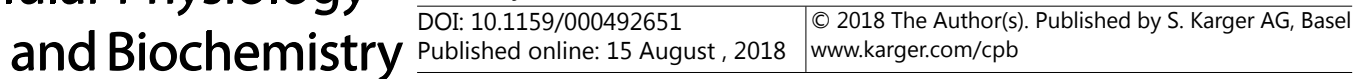 \\ Wang et al.: CD38 Deficiency Suppresses Oxidative Stress}

Fig. 6. The working model for the protection of CD38 deficiency on the lipid-induced oxidative stress in cardiomyocytes. CD38 deficiency increases the intracellular $\mathrm{NAD}^{+}$, and the increased $\mathrm{NAD}^{+}$ as a substrate activates Sirt1 signaling pathway, then inhibiting FASN. Finally, the content of triglycerides in heart was decreased. On the one hand, the increased $\mathrm{NAD}^{+}$also activates Sirt3 which is located in the mitochondria and then promoted the nuclear translocation of FOXO3, resulting in the increases of the expressions of antioxidant/antiapoptotic genes including SOD2 and Bcl2. On the other hand, Sirt3 inhibits the productions of the lipid-overloading induced ROS through directly activating SOD2.

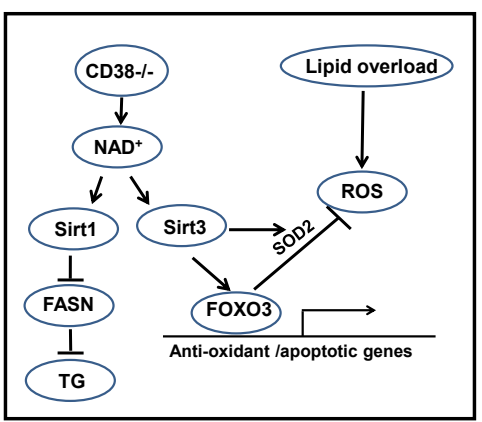

\section{Discussion}

Overweight or obesity, especially abdominal obesity, has become an independent risk factor for many diseases such as cardiovascular diseases, diabetes, multiple types of cancer and renal diseases $[33,34]$. It has been reported that mice consuming a Western diet manifested increased heart and vascular fibrosis which further resulted in cardiac hypertrophy and dysfunction [35]. Individuals with a BMI greater than 30 had more signs of early vascular pathological changes than non-obese people such as coronary arteries dysfunction. Under normal conditions, triglycerides are mainly stored in adipocytes and only a limited triglycerides are stored in the non-fat cells [36]. When adipose tissue fails to store all excess lipids, they will accumulate in other tissues such as muscle, liver and heart. Lipid accumulation in heart was positively correlated with the degree of obesity. In many obese persons, excessive lipid accumulation in and around heart was observed, and the epicardial and intramyocardial lipids were detrimental to cardiac function [37].

It has been reported that CD38 deficiency mice were resistant to high fat diet induced obesity [28]. Our previous data also showed that CD38 deficiency alleviated cardiac function and ROS production in different models. However, whether CD38 could confer protective effects on heart after high fat diet has not been reported. Based on our previous study, we further examined the effects of CD38 deficiency on heart under high fat diet. In the present study, we investigated the metabolic changes in the hearts of CD38 deficiency mice after high fat diet using global metabolomics, a promising qualitative and quantitative analysis approach for all small molecules in biological samples under various stimuli [38]. Our results showed that CD38 deficient hearts exhibited numerous metabolic changes especially in redox homeostasis, nicotinate and nicotinamide metabolism and lipid metabolism compared with wild type. Many studies demonstrated that the imbalance of redox state and oxidative stress was associated with $\operatorname{HFD}[39,40]$. In fact, the increased production of reactive oxygen species, oxidative stress and mitochondrial dysfunctions in heart and other tissues were observed in obesity and obesity-associated metabolic diseases [41, 42]. In the present study, we found that CD38 deficiency significantly decreased oxidative stress under HFD, evidenced by the increased reduced glutathione (GSH) and decreased oxidized glutathione (GSSG) levels, in which the GSH/GSSG ratio was significantly increased in CD38 deficient hearts compared with WT. GSH is an important antioxidant as its thiol group can be oxidized to GSSG by reactive oxygen species. The intracellular GSH/GSSG ratio is considered to be a valid index of oxidative stress. Our results indicated that the increase of the GSH/ GSSG ratio in CD38 deficient hearts was one of the mechanisms for decreasing oxidative stress induced by HFD.

It is well-established that high concentrations of free fatty acids or cholesterol in blood are risk indicators for some diseases such as coronary heart disease and diabetes [43, 44]. Furthermore, it has been reported that an impaired ability of the heart to oxidize excess lipids resulted in an accumulation of intra-myocardial fatty acid metabolites including triglyceride, long chain acyl CoA, ceramide and diacylglyerol (DAG), nevertheless, lowering these harmful 
lipid metabolites can improve cardiac dysfunction [45, 46]. Our study using metabolomic analysis also showed that most of the free fatty acids were reduced while kinds of carnitine were elevated, as well as the key metabolic intermediate acetyl coenzyme A was increased almost 15 folds in heart tissue from CD38 deficient mice, highlighting the importance of lipid metabolism in CD38-mediated resistance to lipid-induced oxidative stress in hearts.

$\mathrm{CD} 38$ is a major $\mathrm{NAD}^{+}$hydrolase in mammals which can use $\mathrm{NAD}^{+}$as a substrate to generate nicotinamide and an ADP-ribosyl product. Microarray data revealed that CD38 was highly expressed in adipose tissues in obese people $[47,48]$. In addition, many studies have also shown that CD38 expression is a reliable negative prognostic marker for chronic lymphocytic leukemia (CLL) patients [49-51]. Therefore, the overexpression of CD38 is correlated with many human diseases. It has been reported that CD38 deficiency increased autophagy [52], while autophagy is closely related to cardiac diseases. In the present study, we found that the intracellular concentration of $\mathrm{NAD}^{+}$in hearts from $\mathrm{CD} 38$ deficient mice was 177 folds higher than wild type mice, which was consistent with our previous study in CD38 knockout or knockdown models [7, 53]. In addition, the intermediate nicotinamide mononucleotide was increased by approximately 6 folds while the nicotinamide and ADPR, the metabolites of CD38, were significantly reduced in CD38 deficient heart under treated with HFD. The canonical role of $\mathrm{NAD}^{+}$is to facilitate hydrogen transfer in key metabolic pathways. Conversion of $\mathrm{NAD}^{+}$to NADH is also important for oxidation of fatty acids and amino acids in mitochondria. Therefore, the increased $\mathrm{NAD}^{+}$promoted the utilization of $\mathrm{NAD}^{+}$for lipid metabolism in CD38 deficient heart, attenuating HFD-induced oxidative stress. In addition, increased NAD ${ }^{+}$also activates the Sirtuins family, such as Sirt1 and Sirt3, the $\mathrm{NAD}^{+}$-dependent deacetylases [14,54], which deacetylate kinds of target genes involved in anti-oxidative stress pathway. To confirm the protective effects of CD38 deficiency on lipid-induced injury in cardiomyocytes, a CD38 knockdown H9C2 stable cell line was used to elucidate the mechanisms. Our results showed that CD38 knockdown significantly increased cell survival after the cells treated with OA. Moreover, CD38 knockdown also improved OAinduced oxidative stress in H9C2 cells, manifested by decreased LDH activity and increased SOD activity. In addition, we also observed that ROS production was significantly increased when cells were treated with oleic acid, while CD38 knockdown reduced its generation compared with control group. These findings indicated that CD38 deficiency alleviated lipidinduced oxidative stress in vivo and in vitro.

Sirt3 pathway was one of the most important pathways for protecting cells against oxidative stress $[26,55]$. It has been reported that Sirt3-deficient cardiomyocytes had high levels of ROS and Sirt3 reduced doxorubicin-induced ROS production mainly via regulating the key antioxidant SOD2 [55]. In addition, a recent study indicated that Sirt3/SOD2 signaling also played a protective role in mitochondrial oxidative stress induced by bile acid in hepatocytes [27]. The reduced oxidative stress by Sirt3 not only relied on the deacetylation of SOD, but also the deacetylated FOXO3 to protect mitochondria against oxidative damage [56]. Sundaresan et al. reported that Sirt3 induced FOXO3a translocation to the nucleus and augmented FOXO3a-dependent antioxidant genes expression, mainly through upregualtion the activities of MnSOD and Catalase [23]. In the present study, we detected the acetylation level of total protein which indirectly reflected the activity of Sirtuins. And the results showed that oleic acid increased acetylation level while CD38 knockdown reduced it compared with control group, indicating that CD38 knockdown increased Sirtuins activity under OA treatment. Moreover, we also examined the expressions of oxidative stress relative signaling molecules Sirt3, SOD and FOX03. The results also showed that the expressions of Sirt3 and its target genes such as SOD and FOXO3 were upregulated in CD38 knockdown cells with or without OA stimulation. Taken together, these experimental results indicated that CD38 knockdown reduced lipid overload-induced oxidative stress in cardiomyocytes possibly through activating Sirt3/FOXO3 signaling pathway._ENREF_37

NAPDH oxidases (NOXs) are responsible for ROS generation, in which the NOX2 and NOX4 are significantly associated with cardiovascular diseases [57]. Studies showed that NOX2 inhibition could prevent oxidative stress induced by saturated fatty acids in 
cardiomyocytes [22] and NOX4 regulated basal and pathological ROS production [58, 59]. It has been reported that the NOX4 deficiency mice were sensitive to HFD-induced obesity, suggesting its anti-adipogenic role [60]. In the present study, we observed that NOX2 and NOX4 expressions were significantly down-regulated in CD38 knockdown cells after $24 \mathrm{hr}$ OA stimulation, especially NOX4. It was well known that oxidative stress was often accompanied by apoptosis. Our results showed that the anti-apoptotic factor Bcl-2 expression was increased, whereas the proapoptotic factor Bax expression was decreased in CD38 knockdown cells treated with OA, suggesting that the reduced NOX expressions and oxidative stress-induced apoptosis may also partially contribute to the protection of CD38 deficiency on the lipid overload injury in cardiomyocytes.

\section{Conclusion}

Our results from in vivo and in vitro studies demonstrated that CD38 deficiency significantly protected hearts from lipid-induced injury via regulating redox homeostasis, lipid metabolism and apoptosis in myocardial cells through activating Sirt3/FOXO3 signaling pathway. Our findings provide new insights into the elucidation of the mechanisms of lipidinduced oxidative stress and heart injury.

\section{Acknowledgements}

The authors thank Dr. Ying Liu for providing the stable CD38 knockdown H9C2 cell line. We also thank all the members in Dr. Xin's laboratory for their generous help and valuable discussion. We are grateful to the members of the Transgenic Mouse Facility in the Institute of Translational Medicine of Nanchang University for animal housing. This work was supported by the National Natural Science Foundation of China (91639106 and 91339113 to Hong-Bo Xin), the National Basic Research Program of China (2013CB531103 to Hong-Bo Xin and Ke-Yu Deng), and Jiangxi Provincial Department of Science and Technology, China (20142BCB24001 to Ke-Yu Deng).

\section{Disclosure Statement}

The authors declare no competing financial interests.

\section{References}

1 Hafstad AD, Boardman N, Aasum E: How exercise may amend metabolic disturbances in diabetic cardiomyopathy. Antioxid Redox Signal 2015;22:1587-1605.

$>2$ Leichman JG, Aguilar D, King TM, Vlada A, Reyes M, Taegtmeyer H: Association of plasma free fatty acids and left ventricular diastolic function in patients with clinically severe obesity. Am J Clin Nutr 2006;84:336341.

-3 Boudina S, Abel ED: Diabetic cardiomyopathy revisited. Circulation 2007;115:3213-3223.

-4 Mandavia CH, Pulakat L, DeMarco V, Sowers JR: Over-nutrition and metabolic cardiomyopathy. Metabolism 2012;61:1205-1210.

5 Stiefel P, Vallejo-Vaz AJ, Garcia Morillo S, Villar J: Role of the renin-angiotensin system and aldosterone on cardiometabolic syndrome. Int J Hypertens 2011;2011:685238.

-6 Malavasi F, Deaglio S, Funaro A, Ferrero E, Horenstein AL, Ortolan E, Vaisitti T, Aydin S: Evolution and function of the adp ribosyl cyclase/cd38 gene family in physiology and pathology. Physiol Rev 2008;88:841-886. 


\section{Cellular Physiology Cell Physiol Biochem 2018;48:2350-2363 \begin{tabular}{l|l|l} 
and Biochemistry Published online: 15 August, 2018 & $\begin{array}{l}\text { @ } 2018 \text { The Author(s). Published by S. Karger AG, Basel } \\
\text { www.karger.com/cpb }\end{array}$ \\
\hline
\end{tabular}}

Wang et al.: CD38 Deficiency Suppresses Oxidative Stress

7 Graeff R, Liu Q Kriksunov IA, Kotaka M, Oppenheimer N, Hao Q, Lee HC: Mechanism of cyclizing nad to cyclic adp-ribose by adp-ribosyl cyclase and cd38. J Biol Chem 2009;284:27629-27636.

8 Chini EN: Cd38 as a regulator of cellular nad: A novel potential pharmacological target for metabolic conditions. Curr Pharm Des 2009;15:57-63.

-9 Xiong J, Xia M, Yi F, Abais JM, Li N, Boini KM, Li PL: Regulation of renin release via cyclic adp-ribosemediated signaling: Evidence from mice lacking cd38 gene. Cell Physiol Biochem 2013;31:44-55.

10 Guse AH: Cyclic adp-ribose: A novel ca2+-mobilising second messenger. Cell Signal 1999;11:309-316.

11 Rah SY, Kwak JY, Chung YJ, Kim UH: Adp-ribose/trpm2-mediated ca2+ signaling is essential for cytolytic degranulation and antitumor activity of natural killer cells. Sci Rep 2015;5:9482.

12 Magnone M, Bauer I, Poggi A, Mannino E, Sturla L, Brini M, Zocchi E, De Flora A, Nencioni A, Bruzzone S: $\mathrm{Nad}+$ levels control ca2+ store replenishment and mitogen-induced increase of cytosolic ca2+ by cyclic adp-ribose-dependent trpm2 channel gating in human t lymphocytes. J Biol Chem 2012;287:21067-21081.

-13 Santella L: Naadp: A new second messenger comes of age. Mol Interv 2005;5:70-72.

14 Aksoy P, Escande C, White TA, Thompson M, Soares S, Benech JC, Chini EN: Regulation of sirt 1 mediated nad dependent deacetylation: A novel role for the multifunctional enzyme cd38. Biochem Biophys Res Commun 2006;349:353-359.

15 Nogueiras R, Habegger KM, Chaudhary N, Finan B, Banks AS, Dietrich MO, Horvath TL, Sinclair DA, Pfluger PT, Tschop MH: Sirtuin 1 and sirtuin 3: Physiological modulators of metabolism. Physiol Rev 2012;92:14791514.

16 Jin L, Galonek H, Israelian K, Choy W, Morrison M, Xia Y, Wang X, Xu Y, Yang Y, Smith JJ, Hoffmann E, Carney DP, Perni RB, Jirousek MR, Bemis JE, Milne JC, Sinclair DA, Westphal CH: Biochemical characterization, localization, and tissue distribution of the longer form of mouse sirt3. Protein Sci 2009;18:514-525.

17 Lombard DB, Alt FW, Cheng HL, Bunkenborg J, Streeper RS, Mostoslavsky R, Kim J, Yancopoulos G, Valenzuela D, Murphy A, Yang Y, Chen Y, Hirschey MD, Bronson RT, Haigis M, Guarente LP, Farese RV, Jr, Weissman S, Verdin E, Schwer B: Mammalian sir2 homolog sirt3 regulates global mitochondrial lysine acetylation. Mol Cell Biol 2007;27:8807-8814.

18 Koentges C, Bode C, Bugger H: Sirt3 in cardiac physiology and disease. Front Cardiovasc Med 2016;3:38.

19 Ansari A, Rahman MS, Saha SK, Saikot FK, Deep A, Kim KH: Function of the sirt3 mitochondrial deacetylase in cellular physiology, cancer, and neurodegenerative disease. Aging Cell 2017;16:4-16.

20 Pei H, Yang Y, Zhao H, Li X, Yang D, Li D: The role of mitochondrial functional proteins in ros production in ischemic heart diseases. Oxid Med Cell Longev 2016;2016:5470457.

21 Hassan W, Rongyin G, Daoud A, Ding L, Wang L, Liu J, Shang J: Reduced oxidative stress contributes to the lipid lowering effects of isoquercitrin in free fatty acids induced hepatocytes. Oxid Med Cell Longev 2014;2014:313602.

\22 Joseph LC, Barca E, Subramanyam P, Komrowski M, Pajvani U, Colecraft HM, Hirano M, Morrow JP: Inhibition of napdh oxidase 2 (nox2) prevents oxidative stress and mitochondrial abnormalities caused by saturated fat in cardiomyocytes. PLoS One 2016;11:e0145750.

-23 Sundaresan NR, Gupta M, Kim G, Rajamohan SB, Isbatan A, Gupta MP: Sirt3 blocks the cardiac hypertrophic response by augmenting foxo3a-dependent antioxidant defense mechanisms in mice. J Clin Invest 2009;119:2758-2771.

-24 Padmaja Divya S, Pratheeshkumar P, Son YO, Vinod Roy R, Andrew Hitron J, Kim D, Dai J, Wang L, Asha P, Huang B, Xu M, Luo J, Zhang Z: Arsenic induces insulin resistance in mouse adipocytes and myotubes via oxidative stress-regulated mitochondrial sirt3-foxo3a signaling pathway. Toxicol Sci 2015;146:290-300.

25 Rangarajan P, Karthikeyan A, Lu J, Ling EA, Dheen ST: Sirtuin 3 regulates foxo3a-mediated antioxidant pathway in microglia. Neuroscience 2015;311:398-414.

26 Qiu X, Brown K, Hirschey MD, Verdin E, Chen D: Calorie restriction reduces oxidative stress by sirt3mediated sod2 activation. Cell Metab 2010;12:662-667.

27 Chen Y, Qing W, Sun M, Lv L, Guo D, Jiang Y: Melatonin protects hepatocytes against bile acid-induced mitochondrial oxidative stress via the ampk-sirt3-sod2 pathway. Free Radic Res 2015;49:1275-1284.

28 Barbosa MT, Soares SM, Novak CM, Sinclair D, Levine JA, Aksoy P, Chini EN: The enzyme cd38 (a nad glycohydrolase, ec 3.2.2.5) is necessary for the development of diet-induced obesity. FASEB J 2007;21:3629-3639. 


\section{Cellular Physiology Cell Physiol Biochem 2018;48:2350-2363 \begin{tabular}{l|l|l|l} 
DOI: 10.1159/000492651 2018 The Author(s). Published by S. Karger AG, Basel & (c)
\end{tabular} and Biochemistry Published online: 15 August, 2018 www.karger.com/cpb}

Wang et al.: CD38 Deficiency Suppresses Oxidative Stress

29 Ge Y, Jiang W, Gan L, Wang L, Sun C, Ni P, Liu Y, Wu S, Gu L, Zheng W, Lund FE, Xin HB: Mouse embryonic fibroblasts from cd38 knockout mice are resistant to oxidative stresses through inhibition of reactive oxygen species production and ca(2+) overload. Biochem Biophys Res Commun 2010;399:167-172.

- 30 Gan L, Jiang W, Xiao YF, Deng L, Gu LD, Guo ZY, Zhou ZC, Wu D, Xin HB: Disruption of cd38 gene enhances cardiac functions by elevating serum testosterone in the male null mice. Life Sci 2011;89:491-497.

-31 Guan XH, Liu XH, Hong X, Zhao N, Xiao YF, Wang LF, Tang L, Jiang K, Qian YS, Deng KY, Ji G, Fu M, Xin HB: Cd38 deficiency protects the heart from ischemia/reperfusion injury through activating sirt1/foxosmediated antioxidative stress pathway. Oxid Med Cell Longev 2016;2016:7410257.

-32 Guan XH, Hong X, Zhao N, Liu XH, Xiao YF, Chen TT, Deng LB, Wang XL, Wang JB, Ji GJ, Fu M, Deng KY, Xin HB: Cd38 promotes angiotensin ii-induced cardiac hypertrophy. J Cell Mol Med 2017;21:1492-1502.

-33 Jindal A, Brietzke S, Sowers JR: Obesity and the cardiorenal metabolic syndrome: Therapeutic modalities and their efficacy in improving cardiovascular and renal risk factors. Cardiorenal Med 2012;2:314-327.

34 Kovesdy CP, Furth SL, Zoccali C: Obesity and kidney disease: Hidden consequences of the epidemic. Intern Med J 2017;47:134-143.

-35 Whaley-Connell A, Sowers JR: Indices of obesity and cardiometabolic risk. Hypertension 2011;58:991-993.

-36 McGavock JM, Victor RG, Unger RH, Szczepaniak LS: Adiposity of the heart, revisited. Ann Intern Med 2006;144:517-524.

37 Iacobellis G, Corradi D, Sharma AM: Epicardial adipose tissue: Anatomic, biomolecular and clinical relationships with the heart. Nat Clin Pract Cardiovasc Med 2005;2:536-543.

38 Nicholson JK, Lindon JC: Systems biology: Metabonomics. Nature 2008;455:1054-1056.

-39 Niu S, Wang L, He M, Peng Y, Li S: Exendin-4 regulates redox homeostasis in rats fed with high-fat diet. Acta Biochim Biophys Sin (Shanghai) 2015;47:397-403.

-40 Geetha R, Sathiya Priya C, Anuradha CV: Troxerutin attenuates diet- induced oxidative stress, impairment of mitochondrial biogenesis and respiratory chain complexes in mice heart. Clin Exp Pharmacol Physiol 2017;44:103-113.

41 Raza H, John A, Howarth FC: Alterations in glutathione redox metabolism, oxidative stress, and mitochondrial function in the left ventricle of elderly zucker diabetic fatty rat heart. Int J Mol Sci 2012;13:16241-16254

42 Ghosh S, Sulistyoningrum DC, Glier MB, Verchere CB, Devlin AM: Altered glutathione homeostasis in heart augments cardiac lipotoxicity associated with diet-induced obesity in mice. J Biol Chem 2011;286:4248342493.

43 Ohrvall M, Berglund L, Salminen I, Lithell H, Aro A, Vessby B: The serum cholesterol ester fatty acid composition but not the serum concentration of alpha tocopherol predicts the development of myocardial infarction in 50-year-old men: 19 years follow-up. Atherosclerosis 1996;127:65-71.

44 Laaksonen DE, Lakka TA, Lakka HM, Nyyssonen K, Rissanen T, Niskanen LK, Salonen JT: Serum fatty acid composition predicts development of impaired fasting glycaemia and diabetes in middle-aged men. Diabet Med 2002;19:456-464.

-45 Young ME, Guthrie PH, Razeghi P, Leighton B, Abbasi S, Patil S, Youker KA, Taegtmeyer H: Impaired long-chain fatty acid oxidation and contractile dysfunction in the obese zucker rat heart. Diabetes 2002;51:2587-2595.

-46 Zhou YT, Grayburn P, Karim A, Shimabukuro M, Higa M, Baetens D, Orci L, Unger RH: Lipotoxic heart disease in obese rats: Implications for human obesity. Proc Natl Acad Sci U S A 2000;97:1784-1789.

-47 Nair S, Lee YH, Rousseau E, Cam M, Tataranni PA, Baier LJ, Bogardus C, Permana PA: Increased expression of inflammation-related genes in cultured preadipocytes/stromal vascular cells from obese compared with non-obese pima indians. Diabetologia 2005;48:1784-1788.

48 Mutch DM, Tordjman J, Pelloux V, Hanczar B, Henegar C, Poitou C, Veyrie N, Zucker JD, Clement K: Needle and surgical biopsy techniques differentially affect adipose tissue gene expression profiles. Am J Clin Nutr 2009;89:51-57.

49 Del Poeta G, Maurillo L, Venditti A, Buccisano F, Epiceno AM, Capelli G, Tamburini A, Suppo G, Battaglia A, Del Principe MI, Del Moro B, Masi M, Amadori S: Clinical significance of cd38 expression in chronic lymphocytic leukemia. Blood 2001;98:2633-2639.

50 Durig J, Naschar M, Schmucker U, Renzing-Kohler K, Holter T, Huttmann A, Duhrsen U: Cd38 expression is an important prognostic marker in chronic lymphocytic leukaemia. Leukemia 2002;16:30-35. 


\section{Cellular Physiology Cell Physiol Biochem 2018;48:2350-2363 and Biochemistry Published 1159/000492651 \begin{tabular}{l|l} 
DOI 102018 The Author(s). Published by S. Karger AG, Basel \\
www.karger.com/cpb
\end{tabular}}

Wang et al.: CD38 Deficiency Suppresses Oxidative Stress

51 Ibrahim S, Keating M, Do KA, O’Brien S, Huh YO, Jilani I, Lerner S, Kantarjian HM, Albitar M: Cd38 expression as an important prognostic factor in b-cell chronic lymphocytic leukemia. Blood 2001;98:181186.

52 Bao J, Li G, Yuan X, Li PL, Gulbins E: Contribution of p62 to Phenotype Transition of Coronary Arterial Myocytes with Defective Autophagy. Cell Physiol Biochem 2017;41:555-568.

-53 Young GS, Choleris E, Lund FE, Kirkland JB: Decreased cadpr and increased nad+ in the cd38-/- mouse. Biochem Biophys Res Commun 2006;346:188-192.

-54 Canto C, Houtkooper RH, Pirinen E, Youn DY, Oosterveer MH, Cen Y, Fernandez-Marcos PJ, Yamamoto H, Andreux PA, Cettour-Rose P, Gademann K, Rinsch C, Schoonjans K, Sauve AA, Auwerx J: The nad(+) precursor nicotinamide riboside enhances oxidative metabolism and protects against high-fat diet-induced obesity. Cell Metab 2012;15:838-847.

55 Cheung KG, Cole LK, Xiang B, Chen K, Ma X, Myal Y, Hatch GM, Tong Q Dolinsky VW: Sirtuin-3 (sirt3) protein attenuates doxorubicin-induced oxidative stress and improves mitochondrial respiration in h9c2 cardiomyocytes. J Biol Chem 2015;290:10981-10993.

-56 Tseng AH, Shieh SS, Wang DL: Sirt3 deacetylates foxo3 to protect mitochondria against oxidative damage. Free Radic Biol Med 2013;63:222-234.

57 Lassegue B, San Martin A, Griendling KK: Biochemistry, physiology, and pathophysiology of nadph oxidases in the cardiovascular system. Circ Res 2012;110:1364-1390.

58 Weyemi U, Lagente-Chevallier O, Boufraqech M, Prenois F, Courtin F, Caillou B, Talbot M, Dardalhon M, Al Ghuzlan A, Bidart JM, Schlumberger M, Dupuy C: Ros-generating nadph oxidase nox4 is a critical mediator in oncogenic h-ras-induced DNA damage and subsequent senescence. Oncogene 2012;31:1117-1129.

59 Hiraga R, Kato M, Miyagawa S, Kamata T: Nox4-derived ros signaling contributes to tgf-beta-induced epithelial-mesenchymal transition in pancreatic cancer cells. Anticancer Res 2013;33:4431-4438.

60 Li Y, Mouche S, Sajic T, Veyrat-Durebex C, Supale R, Pierroz D, Ferrari S, Negro F, Hasler U, Feraille E, Moll S, Meda P, Deffert C, Montet X, Krause KH, Szanto I: Deficiency in the nadph oxidase 4 predisposes towards diet-induced obesity. Int J Obes (Lond) 2012;36:1503-1513. 